\title{
Characteristics Of Monolithic Multisection Distributed-Bragg-Reflector Master-Oscillator Power-Amplifiers
}

Vasile Tronciu ( $\boldsymbol{\sim}$ tronciu@mail.utm.md)

Technical University of Moldova https://orcid.org/0000-0002-9164-2249

\section{Eugeniu Grigoriev}

Technical University of Moldova

Christof Zink

Ferdinand-Braun-Institut gGmbH

Hans Wenzel

Ferdinand-Braun-Institut gGmbH

\section{Research Article}

Keywords: multisection DBR, MOPA, Bragg grating, travelling wave model

Posted Date: March 8th, 2022

DOI: https://doi.org/10.21203/rs.3.rs-1348963/v1

License: (a) (i) This work is licensed under a Creative Commons Attribution 4.0 International License.

Read Full License 


\title{
Characteristics of monolithic multisection distributed-Bragg-reflector master-oscillator power-amplifiers
}

\author{
Vasile Tronciu • Eugeniu Grigoriev • \\ Christof Zink · Hans Wenzel
}

Received: date / Accepted: date

\begin{abstract}
We report theoretical results on the wavelength stability of a multisection master-oscillator power-amplifier emitting at $1064 \mathrm{~nm}$. We use a traveling wave equation model to calculate the optical output power and spectral maps versus the currents injected into the different sections of the device. The numerical model explains quantitatively the experimental findings, particularly the collapse of the power if the current injected into a control section adjacent to the distributed Bragg reflector laser acting as master oscillator exceeds certain values. We investigate the influence of the reflectivity at the facet of the power amplifier on the laser behavior.
\end{abstract}

Keywords multisection DBR · MOPA · Bragg grating · travelling wave model

\section{Introduction}

Applications like free-space communication, spectroscopy, and nonlinear frequency conversion require devices with spatially diffraction-limited and spectral narrow-band emission at several hundreds of milliwatts or even watts output power [1],[2],[3]. These requirements can be met by monolithically integrated master-oscillator $(\mathrm{MO})$ power-amplifier $(\mathrm{PA})$ devices where the $\mathrm{MO}$

Vasile Tronciu

Department of Physics, Technical University of Moldova, bd. Stefan cel Mare 168, Chisinau MD-2004, Moldova

Tel.: +373-22-509917

E-mail: vasile.tronciu@adm.utm.md

Eugeniu Grigoriev

Department of Physics, Technical University of Moldova, bd. Stefan cel Mare 168, Chisinau MD-2004, Moldova

Christof Zink · Hans Wenzel

Ferdinand-Braun-Institut gGmbH, Leibniz-Institut für Höchstfrequenztechnik, GustavKirchhoff-Str. 4, 12489 Berlin, Germany 
is realized either as a ridge-waveguide (RW) distributed feedback (DFB) laser [1], [4], [5] or distributed-Bragg reflector (DBR) laser [6], [7]. The MO emits a single lateral and longitudinal lasing mode, which is strongly amplified in the RW [1] or tapered amplifiers [6], [4], [5], [7] monolithically integrated with the MO.

Typically, the MOPAs have at least two electrically separated contacts, one for the MO and the other for the PA. However, the PA can disturb the MO by thermal cross-talk or optical feedback, resulting in spatio-temporal instabilities such as self pulsations, longitudinal-multi-mode operation, and a deterioration of the spatial beam properties [8], [9]. In order to decouple MO and PA, an additional section with an electrically separated contact, called preamplifier [5] or control section [10] can be implemented between MO and PA. However, it was found experimentally that such a control section can result in a lasing collapse [10]. In this paper, we reveal theoretically the origin of this phenomenon. We also investigate the influence of the reflectivity at the facet of the power amplifier resulting in an unexpected spectral behavior [10] which can be avoided by implementing a waveguide tilt at the facet [7].

In this paper, we use a time-dependent traveling wave (TW) model taking into account the coupling for forward and backward propagating fields in the Bragg section. In Refs. [4], [8], and [9] a TW model was used which takes into account both axial $(z)$ and lateral $(x)$ directions. Due to the fact that the effort for its numerical solution is rather expensive, we use here a simpler model [11], [12] neglecting lateral field diffraction, treating tapered amplifiers simply as a straight amplifier. It was shown in Ref. [8] that such a simplified model suffices to explain the dynamical behavior qualitatively.

The paper is organized as follows. The device structure and experimental results are given in Section 2. Then, the TW model is described in Section 3. Results of the numerical simulations are presented in Section 4. Finally, conclusions are given in Section 5.

\section{MOPA structure and experimental results}

As mentioned in the Introduction, the multisection MOPA under study, schematically shown in Fig. 1, is similar to the device reported in [10]. The only difference is that we replaced the tapered PA with a straight one. It was shown in Ref. [8] that such a simplification still allows a correct qualitative description. Moreover, we leave the PA section unbiased (current $I_{t} \operatorname{extPA}$ ) so that it is optically pumped to near transparency. The MOPA consists of 5 sections, namely (from the right- to the left-hand sides in Fig. 1): a $1 \mathrm{~mm}$ long DBR section at the rear, a $0.75 \mathrm{~mm}$ long gain section $\mathrm{G}$, a $0.25 \mathrm{~mm}$ long DBR section, a $0.5 \mathrm{~mm}$ long preamplifier or control section $\mathrm{CON}$, and a $3.5 \mathrm{~mm}$ long PA at the front. The total length of the device is $6 \mathrm{~mm}$. The active layer based on three compressively strained InGaAs quantum wells extends over all sections. The intended lasing wavelength is around $1064 \mathrm{~nm}$. Both facets of the device are anti-reflection coated. In the simulation, we set the reflectivity 


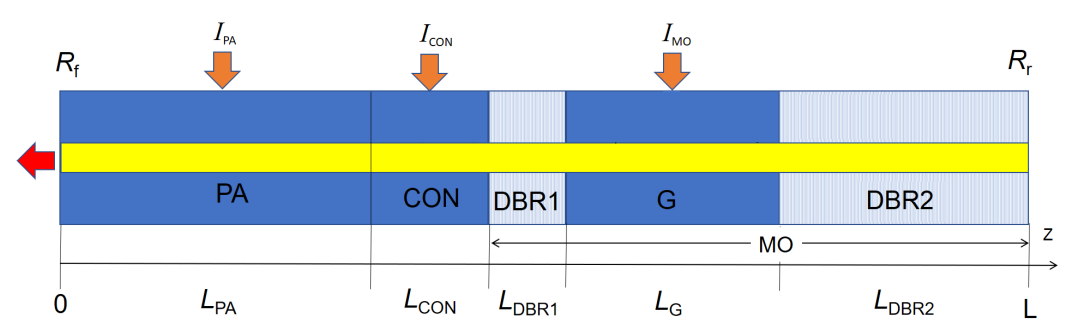

Fig. 1 Schematic view of DBR MOPA device with indicated currents and lengths of the different sections. Throughout the paper, the current $I_{\mathrm{PA}}$ injected into the power amplifier is set to zero.

at the rear facet (DBR1) to zero and varied the intensity reflectivity at the front facet (PA) from 0 to $10^{-2}$.

In what follows, we show for convenience some experimental results already presented in [10]. The power-current characteristic of the MO section measured with a current step of $2 \mathrm{~mA}$ is given in Fig. 2a). Note that the emission propagates through the unbiased $\mathrm{CON}$ and PA sections where some part of the power is absorbed. Lasing starts at a MO current of $I_{\mathrm{MO}}=125 \mathrm{~mA}$. With increasing $\mathrm{MO}$ current, the output power rises nonlinearly with a sawtoothlike shape caused by longitudinal mode jumps as typically observed for DBR lasers [14], [15]. The period of the mode jumps is $\Delta I_{M O} \approx 55 \mathrm{~mA}$ just above threshold and decreases with increasing MO current due to dominating Joule heating which is proportional to the injection current squared. In Fig. 2b) the power-current characteristics in dependence on the CON current are shown for three different $\mathrm{MO}$ currents. The output power increases non-linearly with the CON current up to a maximum value $I_{C O N, M A X}$ depending on the MO current. A further increase of the CON current above $I_{C O N, M A X}$ results in a reduction of the output power until a critical current $I_{C O N, C R I}$ is reached. Above this critical current, no laser emission is observed (lasing collapse). The critical current $I_{C O N, C R I}$ depends on the MO current and the heat sink temperature (not shown here).

\section{Numerical model and parameters}

The numerical model used here is based on the $1 \times 1$ (time $\times$ axial position) dimensional traveling wave equations for the slowly varying complex amplitudes $E^{+}(z, t)$ and $E^{-}(z, t)$ of the counter-propagating optical fields within each section of the device [11],[12]

$$
\frac{n_{\mathrm{g}}}{c_{0}} \frac{\partial}{\partial t} E^{ \pm}(z, t)=\left[\mp \frac{\partial}{\partial z}-i \Delta \beta(N, I)\right] E^{ \pm}(z, t)-i \kappa E^{\mp}(z, t)+F_{\mathrm{sp}}^{ \pm}
$$

where $c_{0}$ is the speed of light in vacuum, $F_{\mathrm{sp}}$ the stochastic contribution of the spontaneous emission, $n_{\mathrm{g}}$ the group index, and $\kappa$ the field coupling coefficient 

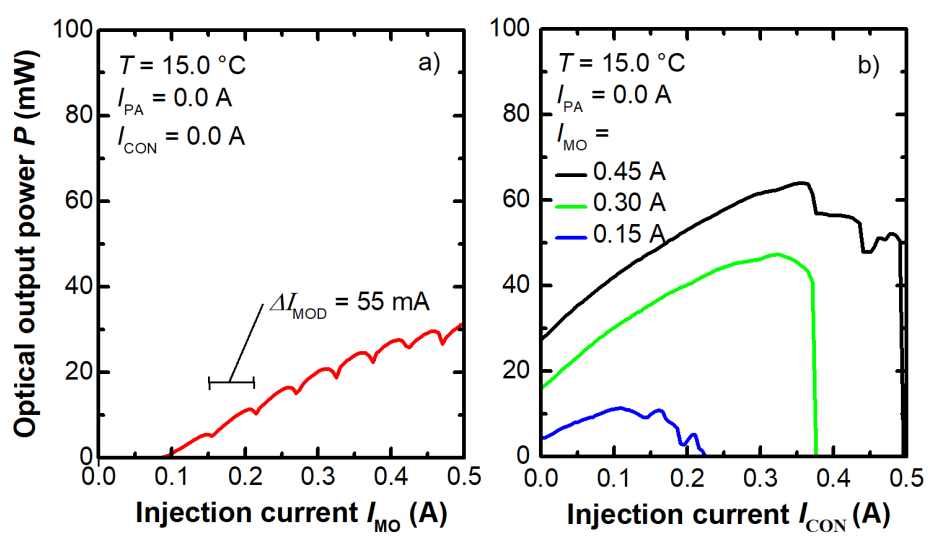

Fig. 2 Experimental results. a) Optical output power in dependence on the MO current. $\mathrm{PA}$ and CON sections are unbiased. b) Optical output power in dependence on the CON current.

due to Bragg gratings. The relative propagation factor in each section is given by

$$
\Delta \beta=\delta_{0}-i \frac{\alpha_{0}}{2}+k_{0}\left[\Delta n_{N}(N)+\Delta n_{T}(I)\right]+i \frac{g(N)-\mathcal{D}}{2},
$$

where $\delta_{0}$ is a static detuning between the sections due to different modal refractive indices, $\alpha_{0}$ the internal optical losses, $k_{0}=2 \pi / \lambda_{0}$ with $\lambda_{0}$ being the reference wavelength, and $\mathcal{D}$ a linear operator modeling gain dispersion. The modal peak gain is assumed to depend logarithmically on the carrier density,

$$
g(N)=\Gamma g^{\prime} N_{\mathrm{tr}} \ln \left(\frac{N}{N_{\mathrm{tr}}}\right)
$$

where $\Gamma$ is the optical confinement factor, $g^{\prime}$ the differential gain, and $N_{\text {tr }}$ the transparency carrier density. The change of the modal index with carrier density is modeled by the square root function [4]

$$
\Delta n_{N}=\tilde{\alpha}_{\mathrm{H}} \frac{\Gamma g^{\prime} N_{\mathrm{tr}}}{k_{0}} \sqrt{\frac{N}{N_{\mathrm{tr}}}} .
$$

The function $\Delta n_{T}(I)$ describes the change of the modal index in a laser section $k$ due to self and cross heating induced by the currents injected in sections $r$ $[4],[14]$,

$$
\Delta n_{T, k}=\frac{n_{\mathrm{g}}}{\lambda_{0}} \sum_{r} C_{k}^{r} \cdot I_{r}, \quad r, k \in[P A, C O N, D B R 1, G, D B R 2],
$$

being the major factor implying transitions between longitudinal modes when the injection current is varied. We mention that in our calculations we consider only the changes of the modal indices induced by currents injected into 
Table 1 Parameters of the standard configuration

\begin{tabular}{llll}
\hline Symbol & Description & Unit & Value \\
\hline$\lambda_{0}$ & reference wavelength & $\mathrm{m}$ & $1.06 \cdot 10^{-6}$ \\
$L_{\mathrm{G}}$ & length of active section & $\mathrm{m}$ & $0.75 \cdot 10^{-3}$ \\
$L_{\mathrm{DBR} 1}$ & length of DBR section & $\mathrm{m}$ & $0.25 \cdot 10^{-3}$ \\
$L_{\mathrm{DBR} 2}$ & length of DBR section & $\mathrm{m}$ & $1.0 \cdot 10^{-3}$ \\
$L_{\mathrm{CON}}$ & length of CON section & $\mathrm{m}$ & $0.5 \cdot 10^{-3}$ \\
$L_{\mathrm{PA}}$ & length of PA section & $\mathrm{m}$ & $3.5 \cdot 10^{-3}$ \\
$R_{\mathrm{r}}$ & rear facet intensity reflectivity & & 0 \\
$R_{\mathrm{f}}$ & front facet intensity reflectivity & & $0 \cdots .0 .01$ \\
$n_{\mathrm{g}}$ & group refractive index & & 3.7 \\
$\kappa$ & coupling coefficient & $\mathrm{m}^{-1}$ & $10 \cdot 10^{2}$ \\
$\alpha_{H}$ & linewidth enhancement factor & & -2.0 \\
$\alpha_{0}$ & internal absorption & $\mathrm{m}^{-1}$ & $2 \cdot 10^{2}$ \\
$\Gamma$ & optical confinement factor & & $2.2 \cdot 10^{-2}$ \\
$g^{\prime}$ & differential gain & $\mathrm{m}^{2}$ & $1450 \cdot 10^{-22}$ \\
$\epsilon_{\mathrm{g}}$ & gain compression factor & $\mathrm{m}^{3}$ & $1 \cdot 10^{-24}$ \\
$N_{\mathrm{tr}}$ & transparency carrier density & $\mathrm{m}^{-1}$ & $1.2 \cdot 10^{-24}$ \\
$d$ & thickness of active layer & $\mathrm{m}^{-9}$ & $7 \cdot 10^{-9}$ \\
$W$ & width of active layer & $\mathrm{m}^{-1}$ & $8 \cdot 10^{-6}$ \\
$A$ & recombination parameter & $s^{-1}$ & $1.7 \cdot 10^{-9}$ \\
$B$ & recombination parameter & $\mathrm{m}^{3} \mathrm{~s}-1$ & $1 \cdot 10^{-16}$ \\
$C$ & recombination parameter & $\mathrm{m}^{6} \mathrm{~s}$ & $40 \cdot 10^{-42}$ \\
$U_{\mathrm{F}}^{\prime}$ & derivative of Fermi level separation & $\mathrm{V} \mathrm{m}^{3}$ & $0.04 \cdot 10^{-24}$ \\
\hline & & &
\end{tabular}

adjacent sections. In this paper, we assume the following values for the selfheating and cross-heating coefficients: $C_{\mathrm{PA}}^{\mathrm{PA}}=0.05 \mathrm{~nm} / \mathrm{A}, C_{\mathrm{CON}}^{\mathrm{CON}}=6.0 \mathrm{~nm} / \mathrm{A}$, $C_{\mathrm{G}}^{\mathrm{G}}=4.0 \mathrm{~nm} / \mathrm{A}, C_{\mathrm{CON}}^{\mathrm{PA}}=C_{\mathrm{PA}}^{\mathrm{CON}}=C_{\mathrm{DBR} 1}^{\mathrm{G}}=C_{\mathrm{DBR} 2}^{\mathrm{G}}=0.35 \mathrm{~nm} / \mathrm{A}, C_{\mathrm{DBR} 1}^{\mathrm{CON}}=$ $1.0 \mathrm{~nm} / \mathrm{A}$.

The rate equation for the carrier density $N(z, t)$ in each section reads

$$
\begin{aligned}
\frac{\partial}{\partial t} N= & \frac{I}{e d W l_{\mathrm{A}}}+\frac{U_{\mathrm{F}}^{\prime}}{e d r_{\mathrm{s}}}(\bar{N}-N)-\left(A N+B N^{2}+C N^{3}\right) \\
& -\frac{c_{0}}{n_{\mathrm{g}}} \Re \sum_{\nu= \pm} E^{\nu *}[g(N)-\mathcal{D}] E^{\nu}
\end{aligned}
$$

where $d$ and $W$ are thickness and width, respectively, of the active region and $A, B$ and $C$ are the recombination parameters. The second term on the right hand side describes the self-distribution of the injected current due to a nonvanishing series resistance $r_{\mathrm{s}}$ with $U_{\mathrm{F}}^{\prime}$ being the derivative of the Fermi level separation and $\bar{N}$ is the average carrier density in the section. For a detailed description of the remaining model equations and parameters, we refer to [11] and [12]. The values of main laser parameters used in our simulations are collected in Table 1.

\section{Results and discussions}

In this section, we present the numerical results obtained using the TW model (1)-(6) incorporated in the software LDSL [13] and the parameters given in 


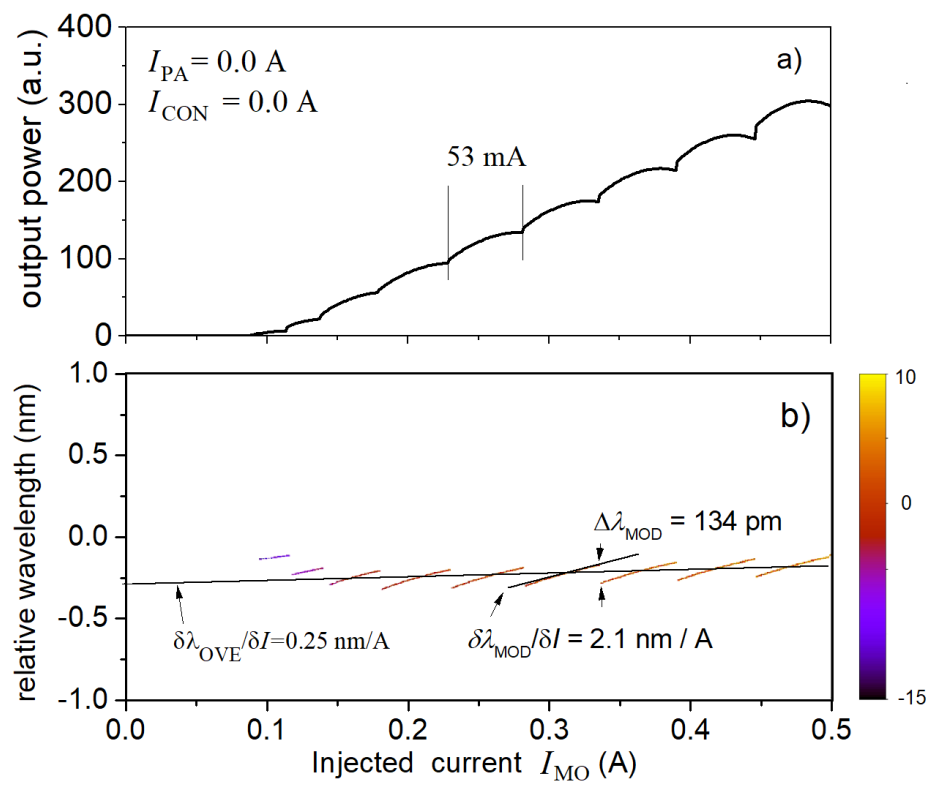

Fig. 3 a) Calculated output power versus current injected into the gain section $G$ of the MO. b) Pseudo-color mapping of the optical spectral densities (in dB) as a function of the MO current and the wavelength (the noisy background is white). The PA and CON sections are unbiased, i.e the PA and CON currents equal zero. Front facet reflectivity is zero.

Table 1. We start our simulations by finding the properties of the MO. With this in mind, the currents injected into CON and PA sections are kept constant equals zero. Figure 3a) shows the dependence of the optical output power at the front facet (at the PA) versus the current injected into the gain section of the MO. The threshold current is $100 \mathrm{~mA}$, similar to the one obtained in the experiment (see Fig. 2a). When the MO current is increased, the output power rises with the typical sawtooth like shape caused by the longitudinal mode jumps as already discussed (see Fig. $3 \mathrm{~b}$ )). The period of the mode jumps is $\Delta I_{\mathrm{MO}} \approx 53 \mathrm{~mA}$, similarly as observed in the experiment (Fig. 2).

Figure $3 \mathrm{~b}$ ) shows a mapping of the optical spectra versus the same range of injection currents as in Fig. 3a). The nearly periodic jumps from a longer wavelength mode to a shorter wavelength mode $\left(\Delta \lambda_{\mathrm{MOD}} \approx 0.134 \mathrm{~nm}\right)$ lead to the modulation of the optical power seen in Fig. 3a). Besides the mode jumps, there is also a variation of the overall lasing wavelength, which is mainly determined by the peak of the reflectivity of the DBR section. Just above threshold, the overall wavelength decreases caused by the optical generation of charged carriers in the DBR section resulting in a decrease of the modal index. For larger currents, the heating of the DBR section (given by the parameters $\left.C_{\mathrm{DBR} 1}^{\mathrm{G}}=C_{\mathrm{DBR} 2}^{\mathrm{G}}\right)$ dominates resulting in a shift of the overall wavelength to larger values. Within a period between the mode jumps, the wavelength of each mode increases with rising current determined by $C_{\mathrm{G}}^{\mathrm{G}}$. For larger currents, the 


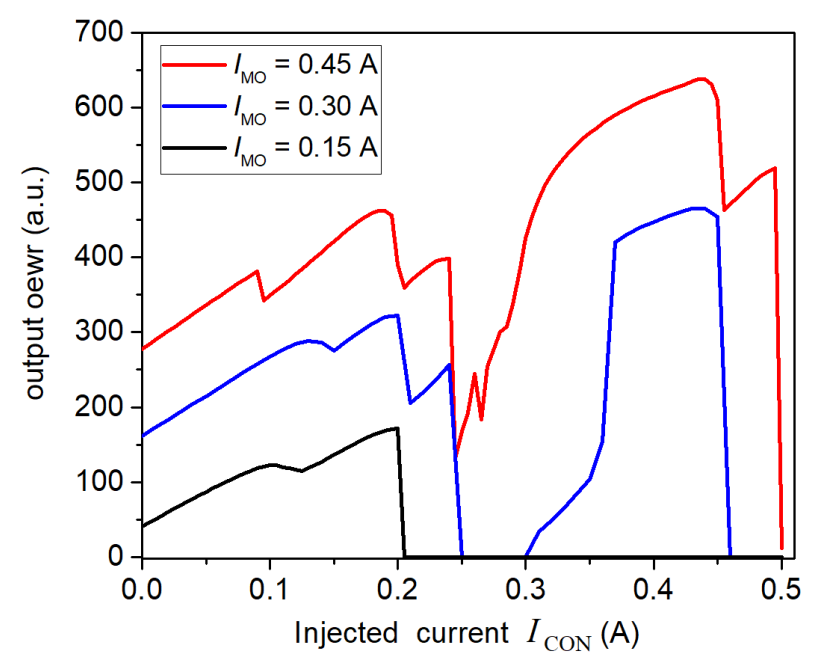

Fig. 4 Output power versus current injected into the control section for different MO currents as indicated in the legend. Other parameters are as in Fig. 3. Front facet reflectivity is zero.

corresponding slope is $\delta \lambda_{\mathrm{MOD}} / \delta I=2.1 \mathrm{~nm} / \mathrm{A}$ and the slope of the shift of the overall wavelength is $\delta \lambda_{\mathrm{OVE}} / \delta I=0.25 \mathrm{~nm} / \mathrm{A}$. These values as well as the spacing of the longitudinal modes (given $\Delta \lambda_{\mathrm{MOD}}$ ) are similar to those of Ref. [10].

As mentioned above, until now the PA and CON sections were unbiased. In what follows we consider what happens if the current injected into CON section, i.e. the preamplifier current, is varied, still keeping PA unbiased. The black line in Fig. 4 shows the dependence of the power on the CON current for a fixed MO current of $0.15 \mathrm{~A}$. The output power rises from $45 \mathrm{~mW}$ to a maximum value of $170 \mathrm{~mA}$ for a $\mathrm{CON}$ current of $0.2 \mathrm{~A}$ and exhibits a kink a $I_{\mathrm{CON}} \approx 110 \mathrm{~mA}$. A further increase of the CON current beyond $0.2 \mathrm{~A}$ leads to drop of the power. No more laser emission is observed (lasing collapse), similarly as in the experiment (Fig. 2). For $I_{\mathrm{MO}}=0.3 \mathrm{~A}$ a similar behavior can be stated, but between CON currents of $0.3 \mathrm{~A}$ and $0.45 \mathrm{~A}$ again lasing operation occurs (blue line of Fig. 4). The power varies between $160 \mathrm{~mW}$ and $460 \mathrm{~mW}$. For a MO current of $0.45 \mathrm{~A}$ the output power rises from $280 \mathrm{~mW}$ followed by jumps and nonlinearities up to a maximum power of $640 \mathrm{~mW}$ at a CON current of $0.45 \mathrm{~A}$ (red line in Fig. 4). The output power drops to zero at a CON current of $0.5 \mathrm{~A}$.

Figure 5 shows a mapping of the optical spectra in dependence on the CON current for the same MO currents as in Fig. 4. For a MO current of $0.15 \mathrm{~A}$, a single jump to a longer wavelength mode occurs at a CON current of 0.125 A. For MO current of $0.3 \mathrm{~A}$, the spectra show different jumps to longer and shorter wavelength modes. One can also see the non lasing region where no lasing modes, but only noise background (white) exists. For all CON currents, single-mode operation can be observed. If the MO current is further increased 


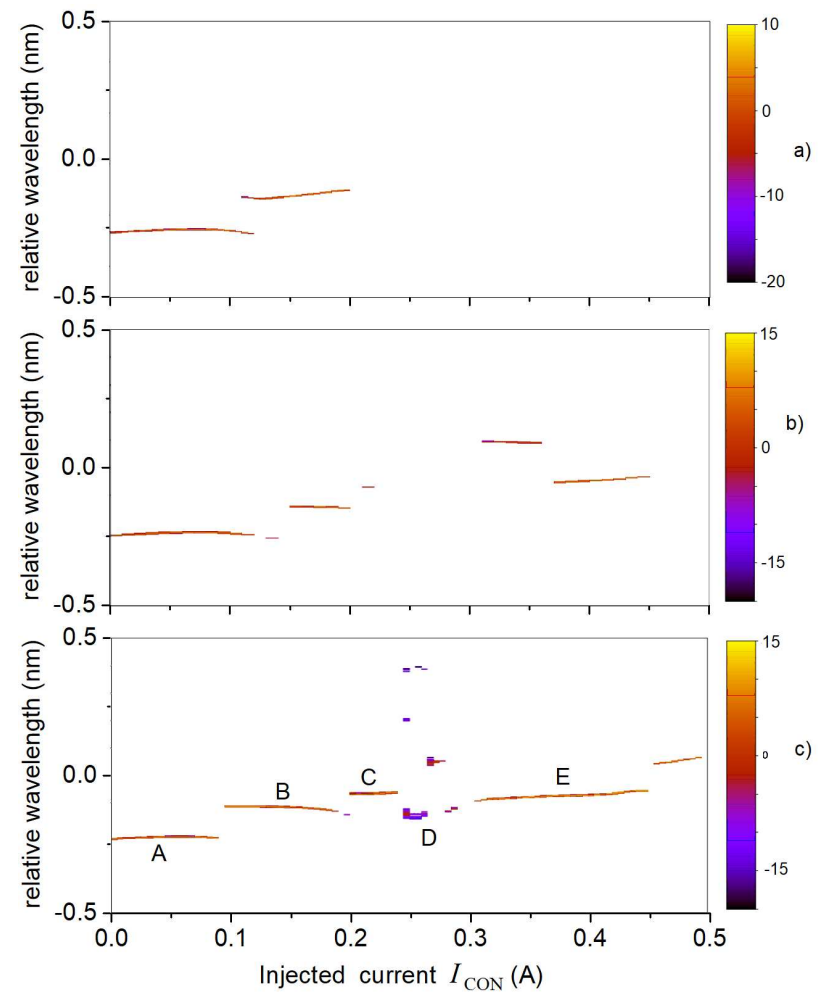

Fig. 5 Pseudo-color mappings of the optical spectral densities (in dB) as a function of the current injected into the CON section and the wavelength for the same parameters and MO currents as in as in Fig. 4. a) $I_{\mathrm{MO}}=0.15 \mathrm{~A}$, b) $I_{\mathrm{MO}}=0.30 \mathrm{~A}$, c) $I_{\mathrm{MO}}=0.45 \mathrm{~A}$. The noisy background is white.

to $0.45 \mathrm{~A}$, an additional multi-mode region around $0.25 \mathrm{~A}$ appears (see region $\mathrm{D}$ in Fig. 5c). This is confirmed by the optical spectrum shown in Fig. 6D.

To understand the modal behavior, we calculated the reflection spectra of sections DBR1 and DBR2 seen from the gain section $\mathrm{G}$ for fixed CON currents corresponding to regions A-E in Fig. 5c. The results are shown in Fig. 6 together with the optical spectra for each region. In the simulation, we modeled the heating of DBR1 due to the CON current by the parameter $C_{\mathrm{DBR} 1}^{\mathrm{CON}}=$ $1.0 \mathrm{~nm} / \mathrm{A}$, but neglected the corresponding heating of DBR2 $\left(C_{\mathrm{DBR} 2}^{\mathrm{CON}}=0\right)$. This model takes into account the effect that in the experiment, the adjacent DBR1 is stronger heated by the current injected into the CON section than the distant DBR2. The heating of DBR1 results in a detuning of the reflection spectra of DBR1 and DBR2 with increasing CON current which is the root cause of the modal behavior. If DBR1 is heated too strongly by a high CON current, the reflection peaks of DBR1 and DBR2 are too far away from each other so that the threshold gain becomes too high and lasing collapse occurs. 

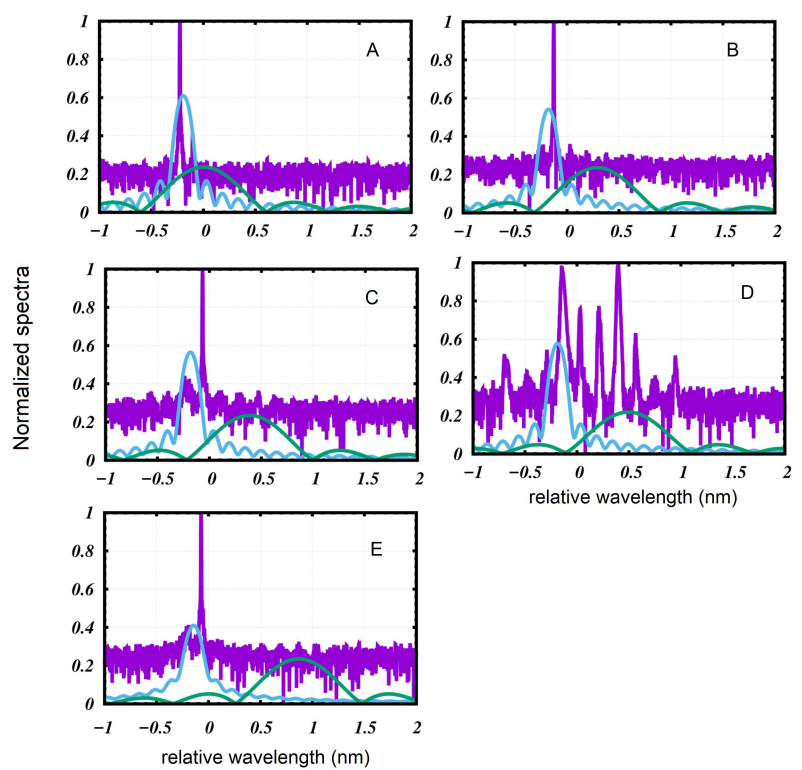

Fig. 6 Optical spectra (purple lines) and reflection spectra of DBR1 (blue) and DBR2 (green) for the different regions of Fig. 5c.

Let us study regions A-E in more detail. In region A (Fig. 6A), the lasing mode is located on the left-hand side of the reflection peak of DBR2. An increase of CON current leads to jumps to longer wavelength modes (regions $\mathrm{B}$ and $\mathrm{C}$ ) on the right-hand side of the reflection peak. The region $\mathrm{D}$ is characterized by multi-mode operation because the reflection peaks of DBR 1 and DBR2 are too distant from each other. Finally, in region E, the mode jumps back to a shorter-wavelength side peak of the reflection spectrum of DBR2.

In what follows, we study the influence of the front facet reflectivity on the output characteristics of the MOPA. We mention that the reflectivity of the front facet can be affected by employing an appropriate coating or tilting the lateral waveguide. First, we reproduce in Fig. 7a) the dependence of the output power on the CON current for a MO current of $0.45 \mathrm{~A}$ and a vanishing reflectivity of the front facet. We plot in this figure like in the following figures the mean (red curve), maximum (blue), and minimum (black) values of the output power collected from pulse traces with a length of $10 \mathrm{~ns}$ at the front facet of the device. If these values calculated for each current coincide, the power can be considered as temporally constant. Otherwise, the laser exhibits dynamic instabilities, as can be seen between mode jumps or for CON currents between 0.25 and $0.3 \mathrm{~A}$ (multi-mode behavior in region $\mathrm{D}$ of Fig. $5 \mathrm{c}$ )). When the front facet reflectivity is increased to $10^{-6}$ and $10^{-4}$, more and more regions of instabilities appear (see Fig. 7 b) and c)). For the highest reflectivity of $10^{-2}$, the device exhibits again a more stable behavior. In this case, the device can 


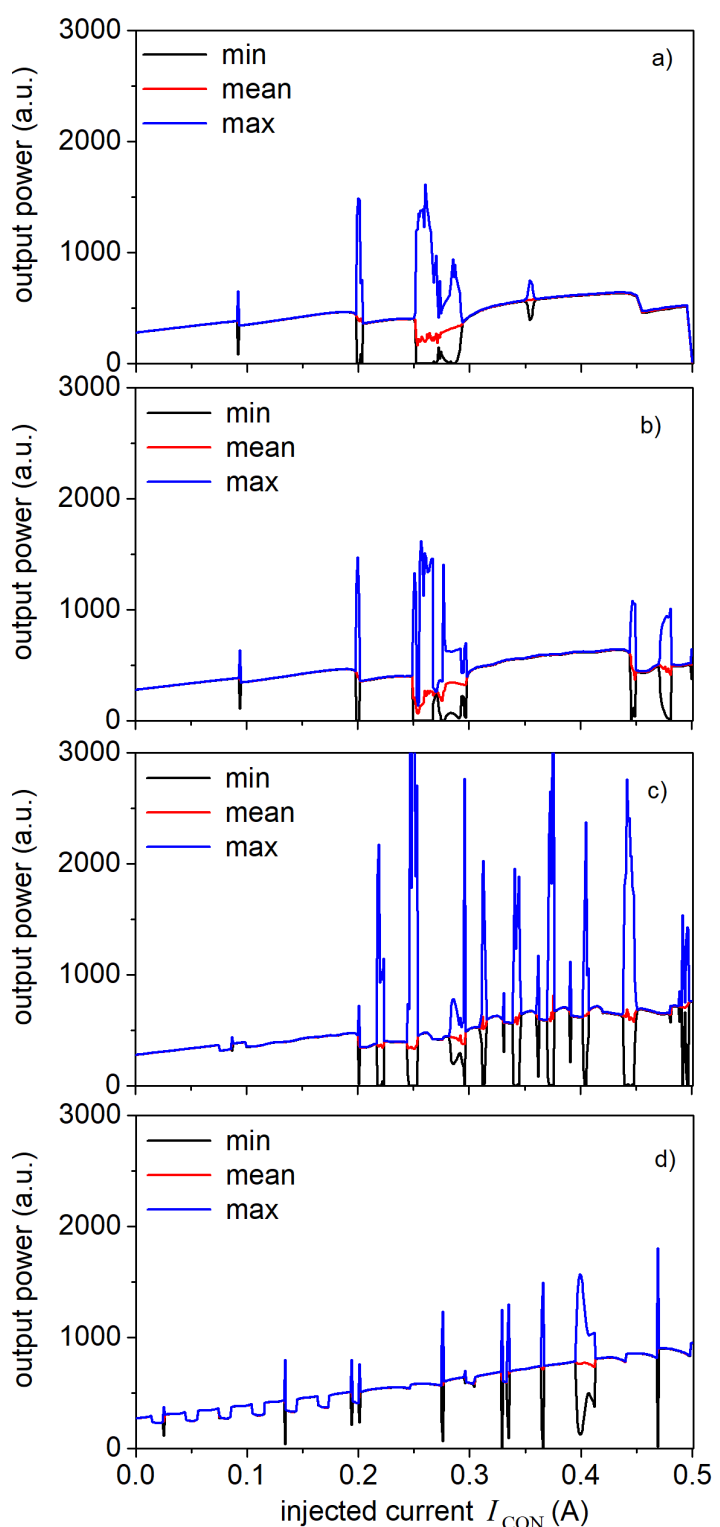

Fig. 7 Output power versus current injected into the control section CON for a) $R_{\mathrm{f}}=0.0$, b) $R_{\mathrm{f}}=10^{-6}$, c) $R_{\mathrm{f}}=10^{-4}$, d) $R_{\mathrm{f}}=10^{-2}$. Other parameters $I_{\mathrm{MO}}=0.45 \mathrm{~A}, I_{\mathrm{PA}}=0$.

be considered to be multi-section laser and not a MOPA because the feedback is mainly provided by DBR2 at the rear and the front facet. 


\section{Summary}

We presented numerical investigations of the behavior of monolithic DBR MOPAs. We used the traveling wave model adapted to the specific multisection device. We were able to reproduce and explain the experimentally observed non-linear dependence of the optical power on a preamplifier or control current and the lasing collapse caused by a thermal detuning of both DBR sections. The numerical simulations show a transition between MOPA and laser types of operation if the front facet reflectivity is increased. For a stable MOPA operation, a front facet reflectivity of $10^{-6}$ or less is needed, which can be achieved by tilted PA [7]. We believe that our work provides a good basis for more detailed investigations of stable operating DBR MOPA devices.

Acknowledgements Part of this work was supported by the National Agency for Research and Development of Moldova within the project 20.80009.5007.08 "Study of optoelectronic structures and thermoelectric devices with high efficiency". We are indebted to M.Radziunas (WIAS Berlin) for his support in using the LDSL tool.

\section{References}

1. Brox, O., Wiedmann,J., Scholz, F., Bugge, F., Fricke, J., Klehr, A., Laurent, T., Ressel, P., Wenzel, H., Erbert G. and Tränkle G.: Integrated 1060nm MOPA pump source for high-power green light emitters in display technology. Proc. SPIE 6909, 69091G (2008)

2. Jensen, O. B., Andersen, P. E., Sumpf, B., Hasler, K. H., Erbert, G. and Petersen, P. M.: 1.5 W green light generation by single-pass second harmonic generation of a singlefrequency tapered diode laser. Opt. Express 17(8), 6532-6539 (2009)

3. Crump, P., Brox, O., Bugge, F., Fricke, J., Schultz, C., Spreemann, M., Sumpf, B. Wenzel, H. Erbert, G.: High power, high efficiency monolithic edge-emitting GaAs-based lasers with narrow spectral widths. in: Coleman, J. J., Bryce, A. C., Jagadish, C. (Eds.): Advances in Semiconductor Lasers (Academic Press, 2012), 49-91 (2012)

4. Spreemann, M., Lichtner, M., Radziunas, M., Bandelow, U., Wenzel, H.: Measurement and simulation of distributed-feedback tapered master-oscillator power amplifiers. IEEE J. Quantum Electron. 45(6), 609-616 (2009)

5. Jedrzejczyk, D., Brox, O., Bugge, F., Fricke, G., Ginolas, A., Paschke, K., Wenzel H., Erbert G.: High-power distributed-feedback tapered master-oscillator power amplifiers emitting at $1064 \mathrm{~nm}$. Proc. SPIE 7583, 758317 (2010)

6. Wenzel, H., Paschke, K., Brox, O., Bugge, F., Fricke, J., Ginolas, A., Knauer, A., Ressel, P., Erbert, G.: $10 \mathrm{~W}$ continuous-wave monolithically integrated master-oscillator poweramplifier. Electron. Lett. 43, 160-161 (2007)

7. Zink, C., Maaßdorf, A., Fricke, J., Ressel, P., Sumpf, B., Erbert, G., Tränkle G.: Monolithic Master Oscillator Tilted Tapered Power Amplifier Emitting 9.5 W at $1060 \mathrm{~nm}$. IEEE Photonics Technology Letters 32, 59 (2020)

8. Mindaugas, R., Tronciu, V., Bandelow, U., Lichtner. M., Spreemann, M., Wenzel, H.: Mode transitions in distributed-feedback tapered master-oscillator power-amplifier: theory and experiments. Opt Quant Electron. 40, 1103-1109 (2008)

9. Tronciu, V., Lichtner, M., Radziunas, M., Bandelow, U., Wenzel, H.: Improving the stability of distributed-feedback tapered master-oscillator power-amplifiers. Opt Quant Electron. 41, 531-537 (2009)

10. Zink, C., Maaßdorf, A., Fricke, J., Ressel, P., Maiwald, M., Sumpf, B., Erbert, G., and Tränkle G.: Diffraction limited $1064 \mathrm{~nm}$ monolithic DBR-master oscillator power amplifier with more than $7 \mathrm{~W}$ output power. Proc. SPIE 10553, 105531C (2018) 
11. Radziunas, M., Wünsche, H. J.: Multisection lasers: longitudinal modes and their dynamics. in: J. Piprek (Ed.), Optoelectronic Devices, Springer: New York, pp. 121-150 (2005)

12. Radziunas, M.: Traveling wave modeling of nonlinear dynamics in multisection laser diodes', in: J. Piprek (Ed.), Handbook of Optoelectronic Device Modeling and Simulation: Lasers, Modulators, Photodetectors, Solar Cells, and Numerical Methods, Vol. 2, CRC Press, Ch. 31 (2017)

13. A software package LDSL-tool. Longitudinal Dynamics of multisection Semiconductor Lasers. http://www.wias-berlin.de/software/ldsl.

14. Radziunas, M., Hasler, K. H., Sumpf,B., Tien, T. Q., Wenzel, H.: Mode transitions in distributed Bragg reflector semiconductor lasers: Experiments, simulations and analysis. J. Phys. B: Atomic, Molecular and Optical Physics 44(10), 105401 (2011)

15. Tronciu, V., Wenzel, H., Radziunas, M., Reggentin, M., Wiedmann, J., Knigge A.: Investigation of red-emitting distributed Bragg reflector lasers by means of numerical simulations. IET Optoelectronics. 12, 228-232 (2020) 